«Diabète et Nutrition : les débats! »

\title{
Place des inhibiteurs des SGLT2 dans le traitement du patient diabétique de type 2
}

\section{Positioning SGLT2 inhibitors in the management of type 2 diabetes}

\author{
A.-J. Scheen \\ Université de Liège, Service de diabétologie, \\ nutrition et maladies métaboliques, \\ et Unité de pharmacologie clinique, \\ Département de médecine, CHU Liège, \\ Belgique.
}

\begin{abstract}
Résumé
Les inhibiteurs des cotransporteurs sodium-glucose de type 2 (iSGLT2 ; gliflozines) agissent en forçant une glucosurie. Ils améliorent le contrôle glycémique tout en exerçant d'autres effets métaboliques et hémodynamiques potentiellement favorables. L'empagliflozine (EMPA-REG OUTCOME) et la canagliflozine (CANVAS) ont démontré une réduction significative des événements cardiovasculaires majeurs, des hospitalisations pour insuffisance cardiaque et des événements rénaux chez des patients diabétiques de type 2 à haut risque cardiovasculaire. La dapagliflozine (effets favorables préliminaires dans le registre observationnel CVD-REAL) est actuellement évaluée dans l'essai DECLARETIMI 58. Les gliflozines sont positionnées en combinaison avec la metformine ou un sulfamide, en triple thérapie orale, ou en association avec l'insuline basale dans les dernières recommandations internationales. Les gliflozines doivent sans doute occuper une place préférentielle chez les patients avec maladie cardiovasculaire établie, à risque d'insuffisance cardiaque et/ou avec insuffisance rénale légère. Leur utilisation est contreindiquée en cas d'insuffisance rénale plus sévère et d'infections uro-génitales récidivantes. Non encore commercialisés en France, les iSGLT2 n'ont pas pu être inclus dans la prise de position de la Société francophone du diabète (SFD) 2017. Cet article analyse leurs divers positionnements potentiels et préférentiels au vu des données de la médecine factuelle et de l'expérience clinique des pays francophones où ils sont déjà disponibles.
\end{abstract}

Mots-clés : Antidiabétiques oraux - gliflozine - inhibiteur des SGLT2 - insuffisance cardiaque - protection cardiovasculaire - protection rénale.

\section{Summary}

Sodium-glucose cotransporter type 2 (SGLT2) inhibitors (SGLT2i; gliflozins) exert their glucose-lowering effect by enhancing glucosuria. Beyond improving glucose control, they are associated with other metabolic and haemodynamic effects that are potentially beneficial. Empagliflozin (EMPA-REG OUTCOME) and canagliflozin (CANVAS) have shown significant reductions in major cardiovascular events, hospitalisation for heart failure and renal outcomes in patients with type 2 diabetes and high cardiovascular risk. Dapagliflozin (preliminary positive effects reported in the observational CVD-REAL registry) is currently evaluated in the DECLARE-TIMI 58 cardiovascular outcome trial. Gliflozins may be used in combination with metformin or a sulphonylurea, in triple oral therapy or in combination with basal insulin according to recent international guidelines. They should most probably be preferred in patients with established cardiovascular disease, at high risk of heart failure and/or with mild renal impairment. Their use is contra-indicated in case of more severe renal insufficiency and in patients with recurrent urinary or genital infections. As they as not commercialized in France yet, SGLT2i were not included in the position statement published by the Frenchspeaking diabetes society (SFD) late 2017. This article analyses the different potential and preferential uses of SGLT2i according to recent data of evidence-based medicine and the clinical experience in French-speaking countries where they are already available.

Key-words: Cardiovascular protection - gliflozin - heart failure - renal protection SGLT2 inhibitor - type 2 diabetes.

\section{André Scheen \\ Département de médecine \\ CHU Sart Tilman (B35) \\ 4000 Liège - Belgique \\ andre.scheen@chu.ulg.ac.be}

C 2018 - Elsevier Masson SAS - Tous droits réservés. 


\section{Introduction}

- Le traitement du diabète de type 2 (DT2) peut désormais faire appel à une grande variété de médicaments antihyperglycémiants, et de nombreux progrès ont été réalisés dans la pharmacothérapie du DT2 au cours des dernières années [1, 2]. Néanmoins, dans toutes les recommandations, la metformine est proposée comme premier choix. Par contre, en cas d'échec de la metformine en monothérapie, diverses solutions peuvent être considérées en ajout à la metformine, sans véritable hiérarchie proposée jusqu'à présent. Une approche personnalisée en fonction des caractéristiques des patients et des médications est généralement mise en avant [3-6]. Parmi les antidiabétiques oraux (ADOs), sachant que les inhibiteurs des alpha-glucosidases (acarbose, miglitol) sont relativement peu utilisés en dehors des pays asiatiques, les sulfamides hypoglycémiants (ou les glinides) sont concurrencés par la pioglitazone (mais retirée du marché en France), les inhibiteurs de la dipeptidyl peptidase-4 (iDPP-4, ou gliptines) et, plus récemment, les inhibiteurs des cotransporteurs sodium-glucose de type 2 (iSGLT2, ou gliflozines). Ces derniers ne sont cependant pas encore commercialisés en France. Ils n'ont donc pas pu être pris en compte dans la prise de position de la Société francophone du diabète (SFD) sur la prise en charge médicamenteuse de l'hyperglycémie du patient DT2, publiée fin 2017 [6]. Pourtant, trois iSGLT2 (canagliflozine, dapagliflozine, empagliflozine) sont déjà commercialisés dans plusieurs autres pays francophones, dont la Belgique, la Suisse et le Québec, comme dans la plupart des pays européens d'ailleurs (tableau I).

- Les iSGLT2 ont un mécanisme d'action unique en inhibant la réabsorption du glucose au niveau tubulaire rénal (figure 1) [7]. Ainsi, ils améliorent le contrôle de la glycémie, à jeun et postprandiale, en forçant une glucosurie et sans induire d'hypoglycémie. Comme cette action est indépendante de l'insuline, que ce soit la réserve insulino-sécrétoire ou la sensibilité des tissus à l'action de l'hormone, l'effet
Tableau I. Inhibiteurs des cotransporteurs sodium-glucose de type 2 évalués dans des études cardiovasculaires et combinaisons fixes développées pour la pratique clinique.

\begin{tabular}{|c|c|c|c|c|}
\hline DCI & $\begin{array}{c}\text { Etude de } \\
\text { sécurité } \\
\text { cardiovasculaire }\end{array}$ & $\begin{array}{c}\text { Nom } \\
\text { commercial }\end{array}$ & $\begin{array}{l}\text { Combinaison } \\
\text { avec la } \\
\text { metformine }\end{array}$ & $\begin{array}{l}\text { Combinaison } \\
\text { avec une } \\
\text { gliptine }\end{array}$ \\
\hline Canagliflozine & CANVAS & Invokana $^{\circledR}$ & Invokamet ${ }^{\circledR}$ & ND \\
\hline Dapagliflozine & $\begin{array}{l}\text { DECLARE-TIMI } \\
58\end{array}$ & Forxiga $^{\circledR}$ & Xigduo $^{\circledR}$ & $\begin{array}{c}\text { Qtern }^{\circledast} \\
\text { (plus saxagliptine) }\end{array}$ \\
\hline Empagliflozine & $\begin{array}{l}\text { EMPA-REG } \\
\text { OUTCOME }\end{array}$ & Jardiance $^{\circledR}$ & Synjardy ${ }^{\circledR}$ & $\begin{array}{c}\text { Glyxambi }^{\circledR} \\
\text { (plus linagliptine) }\end{array}$ \\
\hline
\end{tabular}

$\mathrm{DCl}$ : dénomination commune internationale ; ND : non disponible. anti-hyperglycémiant est remarquale phénotype du patient DT2 (peu - ou pas - de non-répondeurs), pourvu que la fonction rénale soit conservée. Par ailleurs, plus I'hyperglycémie est élevée, plus la glucosurie est importante et plus la réduction du taux d'hémoglobine glyquée $\left(\mathrm{HbA}_{1 \mathrm{c}}\right)$ est marquée, ce qui est également appréciable [7, 8] ; cette différence est plus nette que celle observée avec les autres antidiabétiques, en particulier les iDPP-4. Par ailleurs, la glucosurie entraîne une perte calorique, ce qui provoque une certaine perte de poids (malgré le meilleur contrôle glycémique), à nouveau très reproductible d'une étude à l'autre, et une légère augmentation des concentrations blement reproductible, quel que soit plasmatiques du $\beta$-hydroxybutyrate, considéré comme un meilleur substrat énergétique pour le myocarde et le rein. Il existe aussi une uricosurie, elle-même responsable d'une baisse de l'uricémie. Enfin, ces nouveaux médicaments provoquent également une natriurèse et une diurèse osmotique, avec, comme résultante, une diminution de la pression artérielle et de la surcharge volémique (figure 1) [8, 9]. La démonstration de la sécurité cardiovasculaire (CV) et, si possible, d'une protection vis-à-vis des principales complications CV est devenue une priorité pour les nouveaux traitements proposés pour la prise en charge du DT2 [10]. Les iSGLT2 ont acquis une notoriété évidente depuis les résultats du grand essai CV EMPA-REG

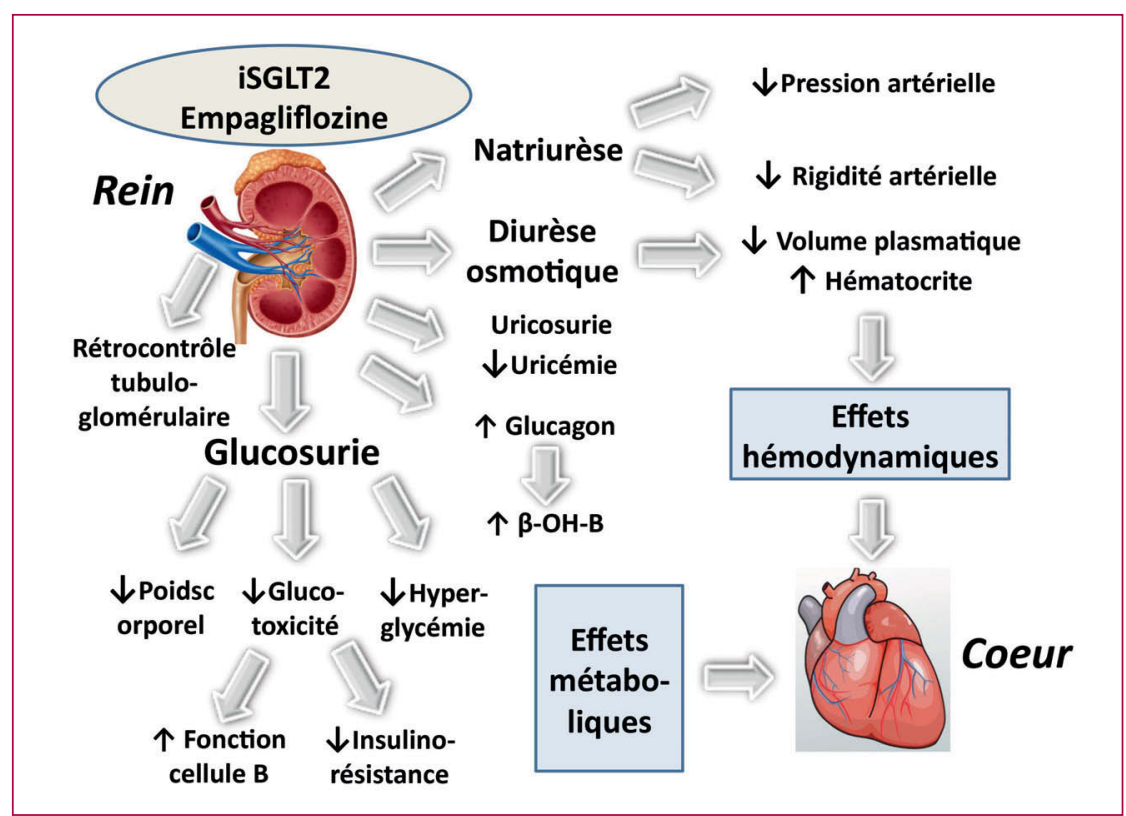

$\beta-\mathrm{OH}-\mathrm{B}$ : bêta-hydroxybutrate.

Figure 1. Effets métaboliques et hémodynamiques des inhibiteurs des cotransporteurs sodium-glucose de type 2 (SGLT2), au-delà de l'amélioration du contrôle glycémique. 
«Diabète et Nutrition : les débats ! "

OUTCOME avec l'empagliflozine [11], confortés récemment par les résultats du programme CANVAS avec la canagliflozine [12] et, dans une moindre mesure, par les données observationnelles de l'enquête CVD-REAL [13], en particulier celles avec la dapagliflozine dans le registre CVD-REAL NORDIC $[14,15]$.

- Le présent article a pour but de décrire les différents positionnements potentiels des iSGLT2 dans la prise en charge du DT2 en analysant, pour chaque positionnement, les éléments positifs et négatifs par rapport aux autres alternatives thérapeutiques. Nous prendrons comme fil conducteur la prise de position de la SFD [6], en y ajoutant la place des iSGLT2 selon notre vision personnelle basée sur les éléments de médecine factuelle disponibles dans la littérature. Nous ferons également mention de quelques manifestations indésirables récemment mises en avant, et rappellerons les précautions d'usage et les principales contre-indications.

\section{En première ligne, comme alternative à la metformine}

- La metformine est le premier choix médicamenteux après échec des mesures hygiéno-diététiques dans les recommandations européennes, américaines, et française [3-6]. Des

\section{Les points essentiels}

- Les inhibiteurs des cotransporteurs sodium-glucose de type 2 (iSGLT2) exercent des effets favorables au-delà de l'amélioration du contrôle glycémique, notamment grâce à la diurèse osmotique et l'augmentation de la natriurèse.

- Sur le plan métabolique, ils diminuent les glycémies à jeun et post-prandiale, sans provoquer d'hypoglycémie, tout en favorisant une perte de poids.

- Comme ces effets sont indépendants de l'insuline, ils sont remarquablement reproductibles (avec peu ou pas de non-répondeurs), tant que la fonction rénale est relativement conservée.

- Sur le plan cardiovasculaire (CV), chez des patients à haut risque, les iSGLT2 réduisent le risque d'événements majeurs, d'hospitalisations pour insuffisance cardiaque, et de mortalité.

- Sur le plan rénal, ils ralentissent la perte de filtration glomérulaire et la progression vers l'insuffisance rénale.

- Les inhibiteurs des iSGLT2 peuvent être utilisés en combinaison avec la plupart des autres médicaments antidiabétiques, mais devraient avoir une place privilégiée chez les patients diabétiques de type 2 à haut risque CV. avec un profil clinique particulier que les iSGLT2 devraient se positionner avantageusement, à savoir les patients obèses hypertendus et, mieux encore, ceux avec antécédents $\mathrm{CV}$ et/ou à risque d'insuffisance cardiaque ou rénale (voir plus loin) (figure 2).

- Si le patient est très déséquilibré au moment du diagnostic, une bithérapie d'emblée est généralement conseillée, en plus du renforcement des mesures hygiéno-diététiques si nécessaire. II faut cependant noter que le niveau d' $\mathrm{HbA}_{10}$ requis pour débuter cette bithérapie initiale peut différer selon les recommandations considérées : en général $9 \%$ (ou $75 \mathrm{mmol} / \mathrm{mol}$ ) $[3,6]$ mais, pour certaines, déjà au-delà de 7,5 \% (ou 57 $\mathrm{mmol} / \mathrm{mol}$ ) [4]. Si l'on doit choisir entre une combinaison metformine + iDPP-4 et une combinaison metformine + iSGLT2, les mêmes critères de choix que ceux qui viennent d'être discutés peuvent s'appliquer $[17,18]$. Des combinaisons fixes avec la metformine ont été développées pour ce qui concerne les différentes gliflozines (tableau l), comme ce fut le cas auparavant pour les gliptines.

\section{En deuxième ligne, en ajout à la metformine}

La question du meilleur choix thérapeutique après échec d'une monothérapie par metformine reste très débattue. Six options possibles sont considérées dans le «position statement " de l'American Diabetes Association (ADA) - European Association for the Study of Diabetes (EASD) de 2015 [3], repris dans le Standard of Care 2017 de l'ADA [19], laissant donc le choix au praticien en fonction des caractéristiques propres à la molécule envisagée et du profil clinique du patient. Dans sa prise de position publiée fin 2017, la SFD a considéré que de nombreux arguments plaident en faveur de l'ajout préférentiel d'un iDPP-4 (figure 2) [6]. Nous avons déjà discuté des arguments plaidant pour un iDPP-4 ou un iSGLT2 $[17,18]$. Tout dépend si le praticien veut privilégier le profil de sécurité, en faveur des iDPP-4, ou le profil d'efficacité, en faveur des iSGLT2 [18]. La décision doit être 
prise en fonction du profil de risque du patient : chez les patients âgés, fragilisés, avec insuffisance rénale modérée à sévère, la préférence sera donnée à une gliptine, tandis que chez les patients à haut risque CV (surtout ceux en prévention secondaire), le choix devra plutôt être orienté vers une gliflozine [20] (figure 2).

\section{En troisième ligne, en ajout à une bithérapie orale}

- Compte tenu de l'histoire naturelle du DT2 et de l'épuisement progressif de la réserve insulino-sécrétoire, beaucoup de patients DT2 finissent par résister à une bithérapie orale. Avant de passer à un traitement injectable, une triple thérapie orale peut être tentée [21, 22]. Plusieurs triples thérapies orales peuvent être envisagées. La prise de position de la SFD a fait mention d'une combinaison metformine + iDPP-4 + un sulfamide hypoglycémiant, ce qui ne paraît cependant pas être la combinaison idéale, de telle sorte que le praticien était plutôt orienté vers le recours à un médicament injectable, soit un agoniste des récepteurs du glucagon-like peptide-1 (AR GLP-1), soit une insuline basale [6]. Lorsque les iSGLT2 seront disponibles en France, ils pourront certainement offrir une solution plus avantageuse pour ce qui concerne une triple thérapie orale (figure 2).

- L'ajout d'un iSGLT2 à une bithérapie orale metformine + un iDPP-4 est une option intéressante compte du fait que les trois molécules agissent par des mécanismes différents et complémentaires [23, 24]. Plusieurs études ont démontré une réduction supplémentaire du taux d' $\mathrm{HbA}_{1 \mathrm{c}}$, sans accroître le risque d'hypoglycémie, accompagnée d'une perte de poids et d'une diminution de la pression artérielle. Les effets de cette combinaison thérapeutique sur les complications micro- ou macrovasculaires ne sont cependant pas connues. Pareille triple thérapie pourrait se faire, soit en ajoutant une gliflozine à une combinaison fixe metformine-gliptine, soit en ajoutant une combinaison fixe gliptine-gliflozine à un traitement par metformine (tableau I).

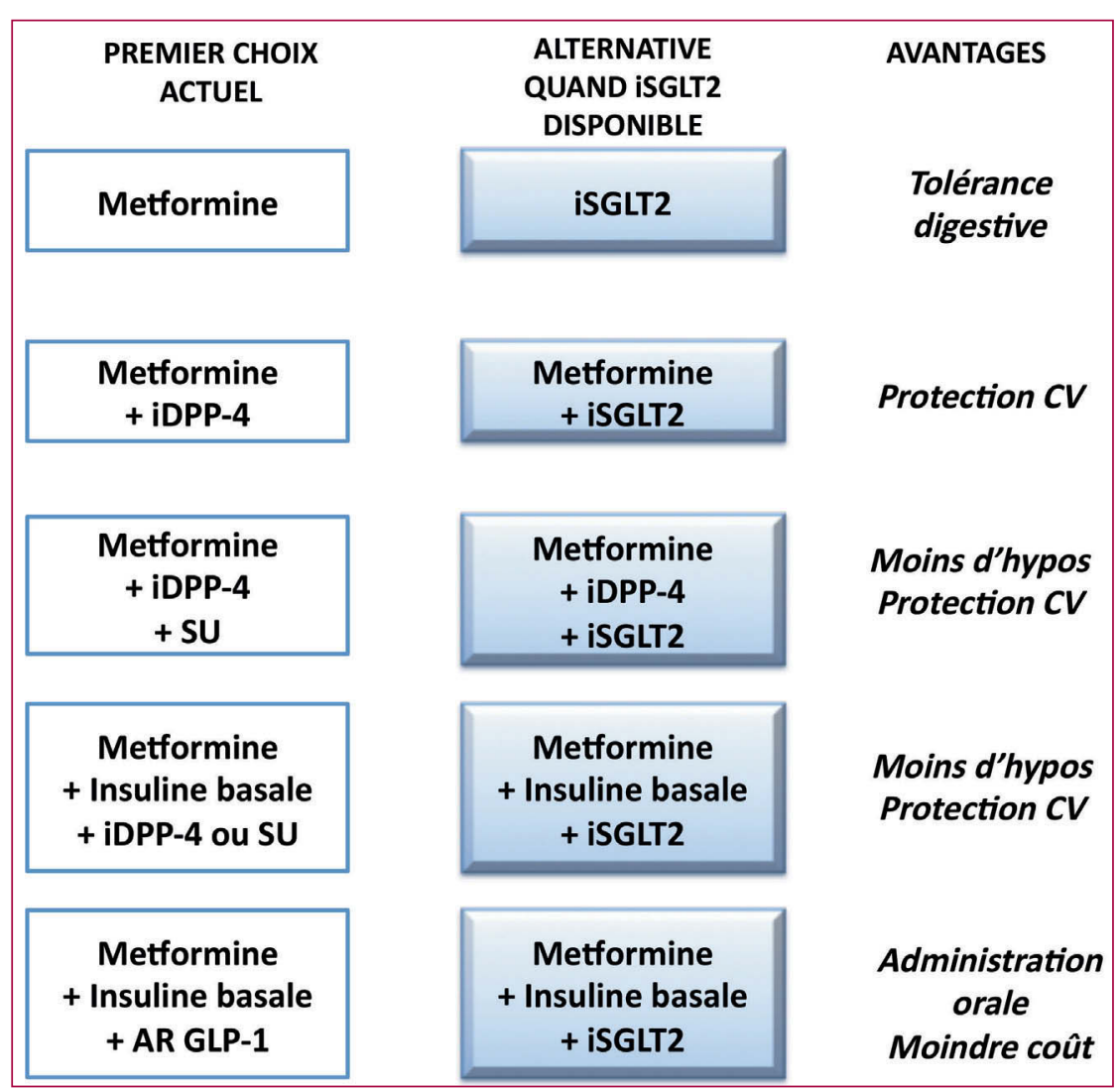

iSGLT2 : inhibiteur des cotransporteurs sodium-glucose de type 2 ; iDPP-4 : inhibiteur de la dipeptidyl peptidase-4 ; SU : sulfamide hypoglycémiant ; AR GLP-1 : agoniste des récepteurs du glucagon-like peptide-1 ; CV : cardiovasculaire ; hypos : hypoglycémies.

Figure 2. Positionnements des inhibiteurs des cotransporteurs sodium-glucose de type 2 comme traitements alternatifs aux solutions thérapeutiques actuellement reconnues dans la prise de position de la Société francophone du diabète (SFD) 2017.

\section{En quatrième ligne, en ajout à une insulinothérapie}

- Dans les recommandations internationales, le passage à l'insuline privilégie une insuline basale. C'est la raison pour laquelle les études ayant testé les iSGLT2 en combinaison avec une insulinothérapie ont recruté des patients traités par une insuline basale (plutôt que des insulines pré-mixées, par exemple) [25]. Pourtant, aussi bien dans EMPA-REG OUTCOME [11] que dans CANVAS [12], près de la moitié des patients recevaient de l'insuline, sans aucune restriction en ce qui concerne le schéma insulinique. Chez la majorité des patients, les ADOs sont maintenus, au moins pour une part, et c'est particulièrement le cas de la metformine, sauf $s$ 'il existe une contre-indication
[26]. Le maintien des sulfamides hypoglycémiants est controversé en raison du risque accru d'hypoglycémie et de prise de poids. Comme rappelé plus haut, les iSGLT2 ne sont pas grevés de ces manifestations indésirables, et leur mécanisme d'action spécifique en font de bons candidats en combinaison avec l'insuline (figure 2).

- Plusieurs études ont démontré l'intérêt d'associer un iSGLT2 à une insulinothérapie basale (+/- metformine) [25]. Une méta-analyse de sept essais cliniques contrôlés a conclu que I'ajout d'un iSGLT2, par comparaison à un placebo, réduit les taux d'HbA $_{1 \mathrm{c}}$ de $0,56 \%$, tout en diminuant les doses quotidiennes d'insuline de $9 \mathrm{UI}$ en moyenne, et sans augmenter le risque d'hypoglycémie, ni le risque d'acidocétose. Par comparaison avec les iDPP-4, les iSGLT2 permettent 
«Diabète et Nutrition : les débats ! "

d'atteindre un meilleur contrôle glycémique, tout en obtenant une plus grande perte de poids et ce, sans augmenter le risque d'hypoglycémie chez des patients DT2 imparfaitement contrôlés sous insuline [27].

- La combinaison d'une insuline basale et d'un AR GLP-1 est une option qui présente divers avantages en termes d'amélioration du contrôle glycémique, de réduction des doses d'insuline, de perte pondérale, de baisse de la pression artérielle et, pour le liraglutide à la dose de 1,8 mg par jour, de protection CV et rénale. Cette association est maintenant mise en avant dans les diverses recommandations internationales [3-5], y compris dans la prise de position de la SFD [6]. En cas de disponibilité des iSGLT2, ceux-ci deviennent de sérieux concurrents pour les AR GLP-1 [28] (figure 2). En effet, ils permettent d'obtenir sensiblement les mêmes effets (même si c'est par des mécanismes différents), avec, comme discuté plus haut, sans doute moins de non-répondeurs, tout en recourant à une simple prise orale plutôt qu'à une injection sous-cutanée, et pour un prix journalier nettement inférieur.

\section{Place des combinaisons orales fixes}

Des combinaisons fixes comprenant un iSGLT2 sont déjà disponibles dans différents pays. Elles concernent la combinaison d'un iSGLT2 avec, soit la metformine, soit un iDPP-4 [29, 30] (tableau I). Les combinaison fixes avec la metformine miment celles déjà sur le marché associant un iDPP-4 avec la metformine. Ces deux types de combinaison fixe sont donc concurrentielles, et visent à promouvoir l'une ou l'autre thérapie en seconde intention en ajout à la metformine, comme discuté plus haut. Par contre, la combinaison fixe d'un iSGLT2 et d'un iDPP-4 se positionne plus tardivement dans l'algorithme, généralement en trithérapie en ajout à la metformine $[23,31]$. Elle pourrait constituer une alternative intéressante à l'introduction d'un AR GLP-1, thérapie injectable et plus onéreuse [28]. Cependant, il n'existe pas d'étude comparative directe entre une combinaison d'un iSGLT2 et d'un iDPP-4, d'une part, et un AR GLP-1, d'autre part.

\section{Inhibiteur des SGLT2 plus un agoniste des récepteurs du GLP-1, une association d'avenir?}

Les iSGLT2 améliorent le pronostic CV et rénal, et un tel effet protecteur a également été rapporté avec certains AR GLP-1. Les mécanismes sous-jacents diffèrent très vraisemblablement selon la classe pharmacologique envisagée, de telle sorte qu'une association des deux approches pourrait s'avérer encore plus efficace [32]. Cette hypothèse reste cependant à démontrer. Une seule étude a été publiée à ce jour démontrant que l'association de la canagliflozine à l'exénatide à libération retardée est plus efficace sur le plan du contrôle glycémique et de certains facteurs de risque [33].

\section{Place éventuelle des gliflozines dans le diabète de type 1}

- Compte tenu du mécanisme d'action princeps rénal des iSGLT2, indépendant de l'insuline [7, 8], il apparaît évident que ces ADOs devraient pouvoir s'avérer aussi utiles chez des patients avec un diabète de type 1 (DT1). Plusieurs études ont déjà été réalisées dans cette population avec des résultats prometteurs, notamment en termes de réduction de la variabilité glycémique [34]. Cependant, le risque d'acidocétose euglycémique (voir ci-dessous) est nettement plus important chez les patients DT1 que chez les patients DT2. Cette complication, potentiellement grave, voire létale, représente incontestablement un frein majeur pour une large utilisation de cette classe thérapeutique chez les patients DT1.

- Même si des résultats plutôt favorables ont été rapportés récemment avec la dapagliflozine dans l'essai DEPICT-1 [35] et avec la sotagliflozine, un inhibiteur des SGLT1 et des
SGLT2, chez des patients DT1 [36], il est important de rappeler que cette indication n'est pas encore acceptée. Des essais similaires ont été réalisés avec les deux autres iSGLT2 déjà commercialisés, la canagliflozine et l'empagliflozine. II conviendra de voir si les résultats de ces essais seront suffisamment convaincants pour que les instances réglementaires acceptent cette nouvelle indication, avec les précautions d'usage pour éviter un risque trop élevé d'acidocétoses.

\section{Rôle particulier dans des populations spéciales}

\section{Prévention secondaire cardiovasculaire}

- Les iSGLT2 ont été étudiés dans de grandes études CV et ont démontré, non seulement la sécurité, mais aussi la supériorité, en comparaison avec un placebo [37, 38].

- La première étude ayant apporté une telle démonstration est l'essai clinique EMPA-REG OUTCOME avec l'empagliflozine, publié en 2015 [11]. Cet essai clinique prospectif a montré que, par rapport au placebo, l'empagliflozine réduit significativement le critère CV composite primaire $(-14 \%)$, mais surtout la mortalité CV (-38\%), la mortalité toutes causes (-32\%), et les hospitalisations pour insuffisance cardiaque (-35\%), sans effet dose (protection comparable avec 10 ou 25 mg d'empagliflozine par jour) (tableau II). Les mécanismes responsables de cette protection, rapide et soutenue, restent controversés [39], même si l'effet diurétique est le plus souvent invoqué, bien que mal étayé [40]. La réduction significative, mais relativement modérée, de la pression artérielle chez des patients DT2 déjà bien contrôlés à l'inclusion, ne paraît pas jouer un rôle déterminant dans l'amélioration du pronostic CV avec les iSGLT2 [41].

- Ces résultats ont été confortés en 2017 par ceux rapportés dans le programme CANVAS avec la canagliflozine [12]. CANVAS a montré une réduction comparable du critère CV composite principal (-14\%) à celle rapportée précédemment dans EMPA-REG OUTCOME 
(tableau II). Par contre, il n'y a pas eu de réduction significative de la mortalité CV, ni de la mortalité globale [12]. Cette différence pourrait être attribuée à la molécule, mais aussi à la population étudiée avec davantage de patients (35\%) en prévention primaire (tableau II). - L'essai clinique CV prospectif avec la dapagliflozine (DECLARE-TIMI 58), dont environ deux tiers des patients DT2 sont en prévention primaire, est toujours en cours, et les résultats ne devraient pas être connus avant 2019.

- En attendant, le registre CVD-REAL, chez des patients DT2 dont une minorité avaient déjà présenté un événement $\mathrm{CV}$, a montré une diminution de la mortalité toutes causes, aussi bien chez des patients traités par canagliflozine (principalement aux États-Unis) que par dapagliflozine (principalement en Europe) par comparaison aux patients DT2 non traités par un iSGLT2 [13]. Ces résultats ont été confirmés plus spécifiquement dans le registre CVD-REAL NORDIC [14], en ce compris dans un sous-groupe de patients DT2 traités par dapagliflozine comparés à des patients DT2 traités par un iDPP-4 [15] (tableau II).

- L'ensemble de ces résultats plaident pour un effet de classe, d'autant plus que des résultats concordants ont été rapportés en ce qui concerne la réduction des hospitalisations pour insuffisance cardiaque (voir ci-dessous) et la diminution des événements rénaux dans EMPA-REG OUTCOME et dans CANVAS (tableau II).

- Convaincues par les résultats d'EMPAREG OUTCOME [11], la Food and Drug Administration (FDA) américaine et l'Agence Européenne du Médicament (EMA) ont reconnu, comme nouvelle indication pour l'empagliflozine, la réduction de la mortalité cardiovasculaire chez des patients avec DT2 et maladie CV [19].

\section{Prévention de l'insuffisance cardiaque}

L'effet favorable sur l'insuffisance cardiaque est remarquablement concordant dans l'étude EMPA-REG OUTCOME avec l'empagliflozine (-35 \%) [11, 42] et dans le programme CANVAS avec la canagliflozine (-33\%) [12] (tableau II).

Tableau II. Résumé des résultats cardiovasculaires dans deux essais contrôlés et dans une étude observationnelle ayant évalué un inhibiteur des cotransporteurs sodium-glucose de type 2 (iSGLT2) avec démonstration d'une protection cardiovasculaire (et rénale).

\begin{tabular}{|c|c|c|c|}
\hline Études & $\begin{array}{c}\text { Essai EMPA-REG } \\
\text { OUTCOME [11] }\end{array}$ & $\begin{array}{l}\text { Programme } \\
\text { CANVAS [12] }\end{array}$ & $\begin{array}{c}\text { Registre CVD- } \\
\text { REAL NORDIC [15] }\end{array}$ \\
\hline $\begin{array}{c}\text { iSGLT2 } \\
\text { versus comparateur }\end{array}$ & $\begin{array}{c}\text { Empagliflozine } \\
10 \text { ou } 25 \mathrm{mg} \\
\text { versus placebo }\end{array}$ & $\begin{array}{c}\text { Canagliflozine } \\
100-300 \mathrm{mg} \\
\text { versus placebo }\end{array}$ & $\begin{array}{l}\text { Dapagliflozine } \\
10 \mathrm{mg} \\
\text { versus iDPP-4 }\end{array}$ \\
\hline $\begin{array}{c}\text { Patients }(n) \\
\text { actif / comparateur }\end{array}$ & 4687 / 2333 & 5795 / 4347 & 10.227 / 30.681 \\
\hline $\begin{array}{c}\text { Patients avec } \\
\text { antécédents CV (\%) }\end{array}$ & 99 & 65 & 23 \\
\hline Suivi (années) & 3,1 & 2,4 & 0,95 \\
\hline $\begin{array}{c}\text { Critère CV composite } \\
\text { primaire* }\end{array}$ & $\begin{array}{c}0,86[0,74-0,99] \\
p<0,001\end{array}$ & $\begin{array}{c}0,86[0,75-0,97] \\
p=0,02\end{array}$ & $\begin{array}{c}0,79[0,67-0,94] \\
p=0,006\end{array}$ \\
\hline $\begin{array}{l}\text { Infarctus du } \\
\text { myocarde }\end{array}$ & $\begin{array}{c}0,87[0,70-1,09] \\
p=0,23\end{array}$ & $\begin{array}{c}0,85[0,69-1,05] \\
\text { NS }\end{array}$ & $\begin{array}{c}0,91[0,72-1,16] \\
p=0,445\end{array}$ \\
\hline AVC ischémique & $\begin{array}{c}1,18[0,89-1,56] \\
p=0,26\end{array}$ & $\begin{array}{c}0,90[0,71-1,15] \\
\text { NS }\end{array}$ & $\begin{array}{c}0,79[0,61-1,03] \\
p=0,086\end{array}$ \\
\hline $\begin{array}{l}\text { Hospitalisation } \\
\text { pour insuffisance } \\
\text { cardiaque }\end{array}$ & $\begin{array}{c}0,65[0,50-0,85] \\
p=0,002\end{array}$ & $\begin{array}{c}0,67[0,52-0,87] \\
p<0,001\end{array}$ & $\begin{array}{c}0,62[0,50-0,77] \\
p<0,001\end{array}$ \\
\hline Mortalité CV & $\begin{array}{c}0,62[0,49-0,77] \\
p<0,001\end{array}$ & $\begin{array}{c}0,87[0,72-1,06] \\
\text { NS }\end{array}$ & $\begin{array}{c}0,76[0,53-1,08] \\
p=0,122\end{array}$ \\
\hline Mortalité globale & $\begin{array}{c}0,68[0,57-0,82] \\
p<0,001\end{array}$ & $\begin{array}{c}0,87[0,74-1,01] \\
\text { NS }\end{array}$ & $\begin{array}{c}0,44[0,33-0,60] \\
p<0,001\end{array}$ \\
\hline $\begin{array}{c}\text { Événements rénaux } \\
\text { composites }\end{array}$ & $\begin{array}{c}0,61[0,53-0,70] \\
p<0,001\end{array}$ & $\begin{array}{c}0,60[0,47-0,77] \\
p<0,001\end{array}$ & ND \\
\hline
\end{tabular}

Résultats exprimés par les « hazard ratio » (avec [intervalles de confiance à $95 \%$ ], et valeurs de $p$ quand disponibles).

ND : non disponible ; NS : non significatif ; AVC : accident vasculaire cérébral ; CV : cardiovasculaire ; iDPP-4 : inhibiteur de la dipeptidyl peptidase-4.

* Mortalité CV, infarctus non mortel, AVC non mortel.

Cet effet a été confirmé dans l'étude observationnelle CVD-REAL, que ce soit dans l'analyse complète des populations européennes et américaines [13] ou dans le registre plus spécifique, mais plus complet, de CVD-REAL NORDIC [14], en ce compris lorsqu'on compare un iSGLT2 et un iDPP-4 [15] (tableau II). Les iSGLT2 représentent la seule classe d'antidiabétiques qui a prouvé un effet favorable sur le risque d'insuffisance cardiaque. En effet, l'autre classe médicamenteuse qui a démontré une protection $\mathrm{CV}$, à savoir les $\mathrm{AR}$ GLP-1 (en particulier, le liraglutide), ont, au mieux, montré un effet neutre sur cette complication [43]. La réduction des hospitalisations pour insuffisance cardiaque dans EMPA-REG OUTCOME a été jugée suffisamment impressionnante pour que la Société européenne de cardiologie reconnaisse l'intérêt de l'empagliflozine chez les patients DT2 pour réduire ou retarder le risque de développer une insuffisance cardiaque (classe de recommandation Ila, niveau d'évidence B) [44].

\section{Protection rénale}

- Les iSGLT2 agissent prioritairement au niveau rénal [7-9]. Compte tenu de leur mécanisme d'action, leur action antihyperglycémiante diminue en fonction de la réduction du débit de filtration glomérulaire (DFG). Dès lors, ils ne doivent pas être instaurés chez les patients avec un DFG inférieur à $60 \mathrm{~mL} / \mathrm{min} / 1,73 \mathrm{~m}^{2}$, et doivent être interrompus si le DFG descend en dessous de $45 \mathrm{~mL} / \mathrm{min} / 1,73 \mathrm{~m}^{2}$. Il est cependant possible que cette restriction évolue avec le temps, car un nombre non négligeable des patients DT2 inclus dans EMPA-REG OUTCOME avaient un DFG entre 30 et $60 \mathrm{~mL} /$ $\mathrm{min} / 1,73 \mathrm{~m}^{2}$ et ces patients ont également bénéficié de la prévention $\mathrm{CV}$ au même titre que ceux avec une fonction rénale normale [45]. 
- Tant EMPA-REG OUTCOME [45] que CANVAS [12] ont montré que, chez des patients DT2 à haut risque $C V$, la prescription d'un iSGLT2 ralentit la perte de filtration glomérulaire au cours du temps et diminue le risque de survenue d'un événement composite rénal, marqueur d'une progression vers l'insuffisance rénale terminale. Ces effets pourraient éventuellement s'expliquer par les effets systémiques des iSGLT2 [9]. Cependant, comme pour le pronostic CV [41], la réduction modeste de la pression artérielle systolique ne paraît pas jouer un rôle majeur dans l'amélioration du pronostic rénal observée avec les iSGLT2 [46]. Un effet intra-rénal, via une restauration du rétrocontrôle tubulo-glomérulaire, est l'explication la plus probante actuellement [9]. Plusieurs essais cliniques centrés plus spécifiquement sur l'évolution de la fonction rénale ou sur la sécurité/efficacité d'emploi chez des patients avec insuffisance rénale sont actuellement en cours et devraient apporter des compléments d'information intéressants.

\section{Précautions d'usage et contre-indications}

Si les iSGLT2 offrent des bénéfices incontestables, en particulier chez les patients DT2 à très haut risque CV, ils sont aussi grevés de certaines manifestations indésirables qui peuvent limiter leur utilisation en pratique clinique [47, 48].

\section{Infections uro-génitales}

Compte tenu du mécanisme d'action des iSGLT2, l'augmentation de la glucosurie s'accompagne d'une augmentation des mycoses génitales, en particulier chez les femmes où le risque est augmenté d'un facteur 3 à 4 [49]. Par contre, le risque d'infections urinaires apparaît très limité [49]. D'un point de vue pratique, il convient de conseiller une hygiène génitale correcte et, sans doute, d'éviter de prescrire un iSGLT2 chez des patient(e)s avec antécédents de mycoses génitales récidivantes. Une méta-analyse récente a montré que l'association d'une gliptine à une gliflozine diminue le risque d'infections génitales [50], en accord avec ce qui avait déjà été suggéré auparavant [23], mais cet effet doit encore être confirmé sur un échantillon plus vaste.

\section{Déshydratation et hypovolémie}

Compte tenu de la diurèse osmotique accompagnée d'une natriurèse, les iSGLT2 peuvent, théoriquement, entraîner un risque de déshydratation et d'événements indésirables en relation avec une hypovolémie, dont une hypotension artérielle, en particulier une hypotension orthostatique [8, 47]. Dans les essais cliniques, force est de reconnaître que ces événements restent relativement exceptionnels. II faut cependant noter que les essais recrutent des patients sélectionnés, et que le risque est sans doute plus élevé dans la vraie vie, englobant des patients plus âgés et fragilisés. En pratique, il est recommandé d'éviter cette pharmacothérapie chez des patients prenant des diurétiques puissants, comme les diurétiques de l'anse. Les iSGLT2 doivent être stoppés en cas d'autres événements susceptibles d'entraîner une déshydratation, comme de la diarrhée et/ou des vomissements, et il convient de se méfier en cas de forte chaleur si une hydratation correcte ne peut être garantie. Leur utilisation dans des périodes de jeûne prolongé avec privation de boisson, comme le Ramadan, n'est pas recommandée pour des raisons assez évidentes, en l'absence d'études validées. En cas de déshydratation importante, de rares cas d'insuffisance rénale fonctionnelle aiguë ont été rapportés [47, 48].

\section{Acidocétose euglycémique}

L'acidocétose diabétique dite euglycémique (en fait, modérément hyperglycémique, avec des taux de glycémie nettement inférieurs à ceux observés dans l'acidocétose diabétique classique en raison de la glucosurie forcée) est certainement la manifestation indésirable qui a suscité le plus de publications les deux dernières années [51-53]. La physiopathologie est complexe, et combine généralement une réduction trop drastique des doses d'insuline, associée à une augmentation des taux de glucagon, une restriction des apports en glucides, et une augmentation des hormones de stress comme dans les suites d'une intervention chirurgicale [52]. Cette complication est rare chez les patients DT2, mais peut survenir plus fréquemment chez les patients DT1 (indication non reconnue actuellement). Si les précautions d'usage sont respectées, le risque de ce type d'acidocétose doit être exceptionnel lors de l'utilisation des iSGLT2 chez les patients DT2 [51].

\section{Fractures osseuses}

Dans le programme CANVAS, un risque accru de fractures osseuses a été rapporté chez les patients DT2 traités par canagliflozine par rapport au groupe placebo [12]. Cette observation a suscité une mise en garde de la part de la FDA et de l'EMA. Les raisons sousjacentes n'apparaissent pas clairement. D'éventuels troubles du métabolisme osseux ne paraissent pas pouvoir expliquer ce risque accru, et la survenue de chutes sur hypotension est une explication alternative qui a été avancée, mais non prouvée.

\section{Amputations}

Dans le programme CANVAS, il a été rapporté davantage d'amputations (la plupart au niveau des orteils, mais certaines plus proximales) dans le groupe traité par canagliflozine par rapport au groupe ayant reçu le placebo [12]. À nouveau, cette observation a été à l'origine d'un avertissement des autorités réglementaires. Cette complication n'a pas été rapportée avec l'empagliflozine dans EMPA-REG OUTCOME [11], ni avec la dapagliflozine dans les essais cliniques de phase 2-3 ou dans les études observationnelles [54]. Aucune explication formelle ne peut être fournie actuellement pour expliquer la survenue de cette complication avec la canagliflozine, ni les apparentes différences avec les molécules de la même classe thérapeutique [55]. Par ailleurs, une étude observationnelle en vie réelle n'a pas confirmé un risque accru d'amputations chez les patients traités par la canagliflozine [56]. En pratique, par prudence, il est déconseillé de prescrire un iSGLT2 chez des patients DT2 avec antécédents d'amputations périphériques ou atteints d'une artériopathie sévère. 


\section{Conclusion}

Les iSGLT2 ont un mécanisme d'action anti-hyperglycémiante innovant qui, in fine, résulte en de multiples effets métaboliques et hémodynamiques susceptibles de protéger les artères, le cœur, et le rein. Ces nouveaux $A D O$ s sont reconnus à part entière dans les différentes recommandations internationales, le plus souvent en combinaison avec d'autres médicaments antidiabétiques, en ce compris I'insuline basale. Au vu des résultats des études CV, ils paraissent particulièrement indiqués chez les patients DT2 avec antécédents $C V$ ou à risque d'insuffisance cardiaque. Un intérêt croissant existe également quant à la protection rénale apportée par cette nouvelle classe thérapeutique, qui n'a certainement pas encore révélé tous ses secrets.

\section{Déclaration d'intérêt}

L'auteur déclare n'avoir aucun conflit d'intérêt en lien avec cet article.

II déclare avoir reçu des honoraires, à titre personnel ou institutionnel, comme orateur, conseiller scientifique et/ou investigateur clinicien de la part des firmes suivantes : AstraZeneca, Boehringer Ingelheim, Eli Lilly, GlaxoSmithKline, Janssen, Merck Sharp \& Dohme, Novartis, Novo Nordisk, Sanofi, Servier ; avoir participé comme investigateur clinicien aux études EMPA-REG OUTCOME avec l'empagliflozine, CANVAS-R avec la canagliflozine, et DECLARE-TIMI 58 et DEPICT-1 avec la dapagliflozine.

\section{Signification des acronymes des études} citées

- CANVAS : CANagliflozin cardioVascular Assessment Study

- CVD-REAL : Comparative effectiveness of cardiovascular outcomes in new users of sodium-glucose cotransporter-2 inhibitors - CVD-REAL NORDIC : Comparative effectiveness of cardiovascular outcomes in new users of sodium-glucose cotransporter-2 inhibitors (Nordic countries: Denmark, Norway, Sweden)

- DECLARE-TIMI 58 : Dapagliflozin Effect on the incidence of CardiovascuLAR EventsTIMI group 58
- DEPICT-1 : Dapagliflozin Evaluation in Patients with Inadequately Controlled Type 1 diabetes

- EMPA-REG OUTCOME : EMPAgliflozin cardiovascular OUTCOME events in type 2 diabetes mellitus patients

\section{Références}

[1] Scheen AJ. Antidiabétiques oraux dans le traitement du diabète de type 2 : perspectives historique et médico-économique. Médecine des maladies Métaboliques 2015;9:186-97.

[2] Scheen AJ. Pharmacological management of type 2 diabetes: what's new in 2017? Expert Rev Clin Pharmacol 2017;10:1383-94.

[3] Inzucchi SE, Bergenstal RM, Buse JB, et al. Management of hyperglycaemia in type 2 diabetes, 2015: a patient-centred approach. Update to a position statement of the American Diabetes Association and the European Association for the Study of Diabetes. Diabetologia 2015;58:429-42.

[4] Garber AJ, Abrahamson MJ, Barzilay JI, et al. Consensus statement by the American Association of Clinical Endocrinologists and American College of Endocrinology on the comprehensive type 2 diabetes management algorithm - 2017 Executive Summary. Endocr Pract 2017;23:207-38.

[5] Qaseem A, Barry MJ, Humphrey LL, et al; Clinical Guidelines Committee of the American College of Physicians. Oral pharmacologic treatment of type 2 diabetes mellitus: a clinical practice guideline update from the American College of Physicians. Ann Intern Med 2017;166:279-90.

[6] Darmon P, Bauduceau B, Bordier L, et al; Société Francophone du Diabète (SFD). Prise de position de la Société Francophone du Diabète (SFD) sur la prise en charge médicamenteuse de l'hyperglycémie du patient diabétique de type 2 . Médecine des maladies Métaboliques 2017;11:577-93.

[7] Abdul-Ghani MA, Norton L, DeFronzo RA Role of sodium-glucose cotransporter 2 (SGLT 2) inhibitors in the treatment of type 2 diabetes. Endocr Rev 2011;32:515-31.

[8] Scheen AJ. Pharmacodynamics, efficacy and safety of sodium-glucose co-transporter type 2 (SGLT2) inhibitors for the treatment of type 2 diabetes mellitus. Drugs 2015;75:33-59.

[9] Heerspink HJ, Perkins BA, Fitchett DH, et al. Sodium glucose cotransporter 2 inhibitors in the treatment of diabetes: cardiovascular and kid ney effects. Potential mechanisms, and clinical applications. Circulation 2016;134:752-72.

[10] Scheen AJ. Historique des études cardiovasculaires : de I'UGDP ... aux dernières études. Médecine des maladies Métaboliques 2017;11(Suppl.1):2S15-2S26.

[11] Zinman B, Wanner C, Lachin JM, et al; EMPAREG OUTCOME Investigators. Empagliflozin, cardiovascular outcomes, and mortality in type 2 diabetes. N Engl J Med 2015;373:2117-28.

[12] Neal B, Perkovic V, Mahaffey KW, et al; CANVAS Program Collaborative Group. Canagliflozin and cardiovascular and renal events in type 2 diabetes. N Engl J Med 2017;377:644-57.

[13] Kosiborod M, Cavender MA, Fu AZ, et al; CVD-REAL Investigators and Study Group. Lower risk of heart failure and death in patients initiated on sodium-glucose cotransporter-2 inhibitors versus other glucose-lowering drugs: the CVD-REAL study (Comparative Effectiveness of Cardiovascular Outcomes in New Users of Sodium-Glucose Cotransporter-2 Inhibitors). Circulation 2017;136:249-59.

[14] Birkeland KI, Jørgensen ME, Carstensen $B$, et al. Cardiovascular mortality and morbidity in patients with type 2 diabetes following initiation of sodium-glucose co-transporter-2 inhibitors versus other glucose-lowering drugs (CVD-REAL Nordic): a multinational observational analysis. Lancet Diabetes Endocrinol 2017;5:709-17.

[15] Persson F, Nyström T, Jørgensen ME, et al. Dapagliflozin is associated with lower risk of cardiovascular events and all-cause mortality in people with type 2 diabetes patients (CVDREAL Nordic) when compared with dipeptidyl peptidase-4 inhibitor therapy: A multinational observational study. Diabetes Obes Metab 2017 Aug 3 [Epub ahead of print].

[16] Palmer SC, Mavridis D, Nicolucci A, et al. Comparison of clinical outcomes and adverse events associated with glucose-lowering drugs in patients with type 2 diabetes: a meta-analysis. JAMA 2016;316:313-24.

[17] Scheen AJ. SGLT2 versus DPP4 inhibitors for type 2 diabetes. Lancet Diabetes Endocrino 2013;1:168-70.

[18] Paquot N, Scheen AJ. Inhibiteur de la DPP-4 ou des SGLT2 après échec de la metformine seule dans le diabète de type 2 . Rev Med Suisse 2017;13:1410-5.

[19] American Diabetes Association. Standards of medical care in diabetes-2017. Diabetes Care 2017;40(Suppl.1):S1-S135.

[20] Scheen AJ, Paquot N. Le choix entre un inhibiteur de la DPP-4 et un inhibiteur des SGLT2 pour traiter le diabète de type 2. Rev Med Liege 2016;71:579-85

[21] Scheen AJ. Pharmacotherapy of 'treatment resistant' type 2 diabetes. Expert Opin Pharmacother 2017;18:503-15.

[22] Scheen AJ. 'Treatment-resistant' type 2 diabetes: Which definition for clinical practice? Diabetes Metab 2017;43:295-7.

[23] Scheen AJ. DPP-4 inhibitor plus SGLT-2 inhibitor as combination therapy for type 2 diabetes: from rationale to clinical aspects. Expert Opin Drug Metab Toxicol 2016;12:1407-17.

[24] Scheen AJ, Paquot N. Combinaison gliptinegliflozine dans le traitement du diabète de type 2. Rev Med Suisse 2016;12:1384-8.

[25] Tang H, Cui W, Li D, et al. Sodium-glucose co-transporter 2 inhibitors in addition to insulin therapy for management of type 2 diabetes mellitus: A meta-analysis of randomized controlled trials. Diabetes Obes Metab 2017;19:142-7.

[26] Scheen AJ, Paquot N. Options thérapeutiques chez un patient diabétique de type $2 \mathrm{mal}$ contrôlé par une insuline basale. Rev Med Suisse 2017;13:1416-20. 
[27] Min SH, Yoon JH, Hahn S, Cho YM. Comparison between SGLT2 inhibitors and DPP4 inhibitors added to insulin therapy in type 2 diabetes: a systematic review with indirect comparison meta-analysis. Diabetes Metab Res Rev 2017;33:e2818.

[28] Gurgle HE, White K, McAdam-Marx C. SGLT2 inhibitors or GLP-1 receptor agonists as second-line therapy in type 2 diabetes: patient selection and perspectives. Vasc Health Risk Manag 2016;12:239-49.

[29] Tan X, Hu J. Empagliflozin/linagliptin: Combination therapy in patients with type 2 diabetes. Ann Endocrinol (Paris) 2016;77:557-62.

[30] Scheen AJ. Dapagliflozin and saxagliptin tablets for adults with type 2 diabetes. Expert Rev Clin Pharmacol 2017;10:1303-16.

[31] Scheen AJ. Pharmacokinetic drug evaluation of saxagliptin plus dapagliflozin for the treatment of type 2 diabetes. Expert Opin Drug Metab Toxicol 2017; 13:583-92.

[32] DeFronzo RA. Combination therapy with GLP-1 receptor agonist and SGLT2 inhibitor. Diabetes Obes Metab 2017;19:1353-62.

[33] Frías JP, Guja C, Hardy E, et al. Exenatide once weekly plus dapagliflozin once daily versus exenatide or dapagliflozin alone in patients with type 2 diabetes inadequately controlled with metformin monotherapy (DURATION-8): a 28 week multicentre, double-blind, phase 3 , randomised controlled trial. Lancet Diabetes Endocrinol 2016;4:1004-16 [Erratum in: Lancet Diabetes Endocrinol 2017;5:e8]

[34] Yang Y, Chen S, Pan H, et al. Safety and efficiency of SGLT2 inhibitor combining with insulin in subjects with diabetes: Systematic review and meta-analysis of randomized controlled trials. Medicine (Baltimore) 2017;96:e6944.

[35] Dandona P, Mathieu C, Phillip M, et al; DEPICT-1 Investigators. Efficacy and safety of dapagliflozin in patients with inadequately controlled type 1 diabetes (DEPICT-1): 24 week results from a multicentre, double-blind, phase 3, randomised controlled trial. Lancet Diabetes Endocrinol 2017:5:864-76 [Erratum in: Lancet Diabetes Endocrinol 2017;5:e8].

[36] Garg SK, Henry RR, Banks P, et al. Effects of sotagliflozin added to insulin in patients with type 1 diabetes. N Engl J Med 2017;377:2337-48.

[37] Wu JH, Foote C, Blomster J, et al. Effects of sodium-glucose cotransporter-2 inhibitors on cardiovascular events, death, and major safety outcomes in adults with type 2 diabetes: a systematic review and meta-analysis. Lancet Diabetes Endocrinol 2016;4:411-9.

[38] Scheen AJ, Ernest P, Jandrain B. Protection cardio-rénale par les inhibiteurs des SGLT2 (gliflozines) : d'EMPA-REG OUTCOME à CANVAS. Rev Med Suisse 2017;13:1421-6.

[39] Scheen AJ. Reduction in cardiovascular and all-cause mortality in the EMPA-REG OUTCOME trial: A critical analysis. Diabetes Metab 2016;42:71-6.

[40] Scheen AJ. Reappraisal of the diuretic effect of empagliflozin in the EMPA-REG OUTCOME : Comparison with classic diuretics. Diabetes Metab 2016;42:224-33.

[41] Scheen AJ. Effects of reducing blood pressure on cardiovascular outcomes and mortality in patients with type 2 diabetes: Focus on SGLT2 inhibitors and EMPA-REG OUTCOME. Diabetes Res Clin Pract 2016;121:204-14.

[42] Fitchett D, Zinman B, Wanner C, et al; EMPAREG OUTCOME $®$ trial investigators. Heart failure outcomes with empagliflozin in patients with type 2 diabetes at high cardiovascular risk: results of the EMPA-REG OUTCOME® trial. Eur Heart J 2016;37:1526-34.

[43] Scheen AJ. GLP-1 receptor agonists and heart failure in diabetes. Diabetes Metab 2017;43(Suppl.1):2S13-2S19.

[44] Ponikowski P, Voors AA, Anker SD, et al; Authors/Task Force Members. 2016 ESC Guidelines for the diagnosis and treatment of acute and chronic heart failure: The Task Force for the diagnosis and treatment of acute and chronic heart failure of the European Society of Cardiology (ESC). Developed with the special contribution of the Heart Failure Association (HFA) of the ESC. Eur Heart J 2016;37:2129-200 [Erratum in: Eur Heart J 2016:ehx158].

[45] Wanner C, Inzucchi SE, Lachin JM, et al; EMPA-REG OUTCOME Investigators. Empagliflozin and progression of kidney disease in type 2 diabetes. N Engl J Med 2016;375:323-34.
[46] Scheen AJ, Delanaye P. Effects of reducing blood pressure on renal outcomes in patients with type 2 diabetes: Focus on SGLT2 inhibitors and EMPA-REG OUTCOME. Diabetes Metab 2017;43:99-109.

[47] Scheen AJ. SGLT2 inhibitors: benefit/risk balance. Curr Diabetes Rep 2016;16:92.

[48] Filippas-Ntekouan S, Filippatos TD, Elisaf MS. SGLT2 inhibitors: are they safe? Postgrad Med 2018;130:72-82.

[49] Li D, Wang T, Shen S, et al. Urinary tract and genital infections in patients with type 2 diabetes treated with sodium-glucose cotransporter 2 inhibitors: A meta-analysis of randomized controlled trials. Diabetes Obes Metab 2017;19:348-55

[50] Fadini GP, Bonora BM, Mayur S, et al. Dipeptidyl peptidase-4 inhibitors moderate the risk of genitourinary tract infections associated with sodium-glucose co-transporter-2 inhibitors. Diabetes Obes Metab 2017 Oct 20 [Epub ahead of print].

[51] Rosenstock J, Ferrannini E. Euglycemic diabetic ketoacidosis: a predictable, detectable, and preventable safety concern with SGLT2 inhibitors. Diabetes Care 2015;38:1638-42.

[52] Qiu H, Novikov A, Vallon V. Ketosis and diabetic ketoacidosis in response to SGLT2 inhibitors: Basic mechanisms and therapeutic perspectives. Diabetes Metab Res Rev 2017;33:e2886.

[53] Fadini GP, Bonora BM, Avogaro A. SGLT2 inhibitors and diabetic ketoacidosis: data from the FDA Adverse Event Reporting System. Diabetologia 2017;60:1385-9.

[54] Jabbour S, Seufert J, Scheen A, et al. Dapagliflozin in patients with type 2 diabetes mellitus: A pooled analysis of safety data from phase IIb/III clinical trials. Diabetes Obes Metab 2017 Sep 26 [Epub ahead of print].

[55] Deeks ED, Scheen AJ. Canagliflozin: a review in type 2 diabetes. Drugs 2017:77:1577-92.

[56] Yuan Z, DeFalco FJ, Ryan PB, et al. Risk of lower extremity amputations in people with type 2 diabetes mellitus treated with sodium-glucose co-transporter-2 inhibitors in the USA: A retrospective cohort study. Diabetes Obes Metab 2017 Sep 12 [Epub ahead of print]. 